\title{
A Signal Processing Approach to Generalized 1-D Total Variation
}

\author{
Fikret Işık Karahanoğlu, İlker Bayram, and Dimitri Van De Ville, Member, IEEE
}

\begin{abstract}
Total variation (TV) is a powerful method that brings great benefit for edge-preserving regularization. Despite being widely employed in image processing, it has restricted applicability for 1-D signal processing since piecewise-constant signals form a rather limited model for many applications. Here we generalize conventional $\mathrm{TV}$ in 1-D by extending the derivative operator, which is within the regularization term, to any linear differential operator. This provides flexibility for tailoring the approach to the presence of nontrivial linear systems and for different types of driving signals such as spike-like, piecewise-constant, and so on. Conventional TV remains a special case of this general framework. We illustrate the feasibility of the method by considering a nontrivial linear system and different types of driving signals.
\end{abstract}

Index Terms-Differential operators, linear systems, regularization, sparsity, total variation.

\section{INTRODUCTION}

$\mathbf{T}$ IKHONOV regularization is one traditional way in engineering and statistics to deal with ill-posed problems; i.e., the solution should provide a balance between the data term (consistency with the measurements) and the regularization term (based on prior information). Generalizations of this scheme employ the energy of the solution's image under a linear operator instead of the energy of the solution [1]. Such regularization strategies typically lead to smooth solutions when employing $\ell_{2}$-norm. Alternatively, $\ell_{1}$-norm regularizations have attracted a lot of attention for their sparsity-promoting property; i.e., they favor sparse representations with many zeros or very small coefficients. The $\ell_{1}$-norm is the relaxed convex counterpart of the ideal sparsity-promoting, but nonconvex, $\ell_{0}$ sparsity count (the number of nonzero components) [2]. A particular

Manuscript received June 23, 2010; revised December 14, 2010, March 21, 2011, June 22, 2011, July 05, 2011, July 12, 2011, and July 14, 2011; accepted July 20, 2011. Date of publication August 12, 2011; date of current version October 12, 2011. The associate editor coordinating the review of this manuscript and approving it for publication was Prof. Namrata Vaswani. This work was supported in part by the Swiss National Science Foundation under grant PP00P2-123438 and in part by Center for Biomedical Imaging (CIBM) of the Geneva-Lausanne Universities and the EPFL.

F. I. Karahanoğlu and D. Van De Ville are with the Medical Image Processing Lab (MIPLAB), Institute of Bioengineering, Ecole Polytechnique Fédérale de Lausanne (EPFL), Lausanne CH-1016, Switzerland, and the Department of Radiology and Medical Informatics, University of Geneva, (e-mail: isik.karahanoglu@epfl.ch; dimitri.vandeville@epfl.ch).

I Bayram was with the Biomedical Imaging Group (BIG) at EPFL. He is now with the Electronics and Telecommunication Engineering Department, İstanbul Technical University (ITU), Istanbul 34469, Turkey (e-mail: ilker.bayram@itu. edu.tr).

This paper has supplementary downloadable multimedia material available at $\mathrm{http}$ ://ieeexplore.ieee.org provided by the authors. This summarizes the sound wave processing examples and includes both graphic and audio files. This material is almost $10 \mathrm{MB}$ in size.

Color versions of one or more of the figures in this paper are available online at http://ieeexplore.ieee.org.

Digital Object Identifier 10.1109/TSP.2011.2164399 case of $\ell_{1}$-norm based regularization, total variation (TV), has been widely applied in image processing [3], including applications like image denoising, restoration and deconvolution [4], [5]. In 2-D, TV is defined as the $\ell_{1}$-norm of the magnitude of the gradient. In this respect, TV regularization preserves edge information and hence offers superior approximation quality for most real-world data compared with (smooth) $\ell_{2}$-based regularization. In a typical denoising problem, the aim is to recover an object $\mathbf{x}$, given its noisy measurements $\mathbf{y}$. If the object $\mathbf{x}$ is known to be sparse in a given representation, this information can be used to come up with a regularization strategy. In this regard, promoting "sparsity" of the analysis or synthesis coefficients leads to two different regularization strategies [6]. More precisely, if $\mathbf{T}$ denotes the synthesis operator of a frame, "synthesis prior" denoising requires the solution $\tilde{\mathbf{x}}$ to satisfy

$$
\tilde{\mathbf{x}}=\mathbf{T} \tilde{\mathbf{c}}, \text { where } \tilde{\mathbf{c}}=\arg \min _{\mathbf{c}}\|\mathbf{y}-\mathbf{T} \mathbf{c}\|_{2}^{2}+\|\mathbf{c}\|_{p}^{p}
$$

where $p=1$ for $\ell_{1}$-constrained fitting regression (see, for example, [7]-[11]). On the other hand, the "analysis prior" formulation looks for the solution of

$$
\tilde{\mathbf{x}}=\arg \min _{\mathbf{x}}\|\mathbf{y}-\mathbf{x}\|_{2}^{2}+\|\mathbf{F} \mathbf{x}\|_{p}^{p}
$$

where $\mathbf{F}$ denotes the analysis operator of the frame. For orthonormal bases, the two approaches yield the same result; for redundant frames, even tight ones, they do not. In this setting, Tikhonov and TV regularized formulations of the denoising problem can be regarded as generalizations (since the associated operators are not necessarily the analysis operators of a frame anymore) of the analysis prior formulation, which rely on the $\ell_{2}$ and the $\ell_{1}$-norm, respectively.

$\mathrm{TV}$ exploits the combination of a derivative operator with the $\ell_{1}$-norm, which makes this regularization an ideal option when dealing with piecewise-constant signals. However, in many 1-D applications much more complex signals are encountered. Piecewise-polynomial signals can be dealt with by TV with built-in higher order derivatives [12]-[16]. For example, TV with the second-order derivative is optimal for piecewise-linear signals. The benefit of adding higher order derivatives is that they reduce the staircase effect of the first order derivative in TV and bring flexibility to the reproduced signal. This extension has been used to retain smooth transitions while keeping sharp edges [16], for texture extraction [17], and has recently been reintroduced for MRI reconstruction [18]. The early application of TV regularization has been hampered by rather high computational complexity, but the recent advances in the field have led to fast algorithms for the $\ell_{1}$ minimization problem. Today, the algorithms at hand range from dual methods [19], iterative reweighted norm (IRN) [20], fixed point algorithm [21], graph cuts [4] to (fast) iterative shrinkage-thresholding algorithm ((F)ISTA), and so on [5], [22], [23]. It is important to 
note that basis-pursuit denoising [7] can offer a "synthesis" counterpart of TV; i.e., inverting the derivative operator leads to a dictionary with (shifted) Heaviside step-functions. Therefore, analysis-prior and synthesis-prior methods seem to be equivalent from a theoretical point-of-view, but the representation by the Heaviside dictionary is unstable. For example, in [24], the analysis counterpart of the constructed dictionary is preferred for its simplicity. In some cases, a specific dictionary can be built for the synthesis operator [25], [26], including generalizations of wavelet design [27], [28].

Here, we extend the conventional TV concept and adapt the regularization for any linear differential operator $L$. The choice of the operator can be tuned to the presence of a linear system and can be adapted to the type of the driving signal that is expected. We propose generalized TV regularization for the typical setting in signal processing dealing with uniformly sampled data. We also provide two practical algorithms to solve the optimization problem at hand.

The paper is organized as follows. We elaborate conventional TV as a mathematical principle and then introduce our generalized TV scheme in Section II. Section III is devoted to the algorithms and how to modify these general-purpose solvers for generalized TV regularization. In Section IV we simulate a system of third-order linear differentials and compare the performance of proposed generalized TV and conventional TV. Finally, we comment on the numerical results and draw some conclusions.

\section{Generalized Total VARIATION}

\section{A. Beyond Conventional TV}

We introduce a new regularization term that is inspired from the combination of TV with linear differential operators. We first revisit conventional $\mathrm{TV}$, which forms the fundamental mathematical concept behind our methodology.

Definition 1 (Total Variation): TV of a continuous-domain function $x(t)$ on interval $[a, b]$, is defined as the supremum of absolute differences for any finite partition $P=\left\{t_{0}=a, \ldots, t_{n}, \ldots, t_{m}=b\right\}_{n}$ :

$$
\operatorname{TV}\{x\}=\sup _{P} \sum_{n=1}^{m}\left|x\left(t_{n}\right)-x\left(t_{n-1}\right)\right| .
$$

If the first derivative of $x(t)$ is well defined, then TV can be shown to be equivalent to

$$
\operatorname{TV}\{x\}=\int_{a}^{b}|D\{x\}(t)| d t
$$

where $D$ is regular (continuous-domain) derivative [4].

For many applications in signal processing, the data is acquired and available on a uniform grid. Specifically, we consider the series of samples $\mathbf{x}=\left[x\left(t_{n}\right)\right]_{n \in \mathbb{Z}}$. In such a case, TV can be computed as the $\ell_{1}$-norm of the finite-difference operator

$$
\operatorname{TV}\{\mathbf{x}\}=\sum_{n \in \mathbb{Z}}|\Delta\{\mathbf{x}\}[n]|
$$

where $\Delta\{\mathbf{x}\}[n]=x\left[t_{n}\right]-x\left[t_{n-1}\right]$. This point of view clearly reveals the link between the formulations (3) and (4); i.e., the finite difference $\Delta$ is the discrete counterpart of the continuous- domain derivative $D$. In fact, in the case of uniform discrete data as will be considered further on, the concept of TV can seamlessly be extended for discrete filters that are associated to general linear differential operators.

Definition 2 (Generalized Total Variation): For a discrete signal $\mathbf{x}$, we define the generalized TV as

$$
\operatorname{TV}\{\mathbf{x}\}=\sum_{n \in \mathbb{Z}}\left|\Delta_{L}\{\mathbf{x}\}[n]\right|
$$

where $\Delta_{L}{ }^{1}$ is the discrete version of the $N$ th-order differential operator

$$
L=\prod_{i=1}^{N}\left(D-\alpha_{i} I\right)\left(\prod_{i=1}^{M}\left(D-\gamma_{i} I\right)\right)^{-1}
$$

with $I$ is the identity operator, $\alpha_{i} \in \mathbb{C}, i=1, \ldots, N$, and $\gamma_{i} \in \mathbb{C}, i=1, \ldots, M$, the zeros and poles of the operator, respectively, and $M<N$. We conveniently characterize the operator by $\boldsymbol{\alpha}=\left(\alpha_{1}, \ldots, \alpha_{N}\right)$ and $\boldsymbol{\gamma}=\left(\gamma_{1}, \ldots, \gamma_{M}\right)$. This operator can also be used to build exponential B-splines [29], [30] and wavelets [27].

Clearly, the definition in (5) includes conventional TV; i.e., the case $N=1$ with $\alpha_{1}=0$ and $M=0$ reverts to that case. It should also be noted that for $M=0$ the discrete operator $\Delta_{L}$ has finite length $(N+1)$. For $M>0$, the support of the discrete operator $\Delta_{L}$ becomes infinite in general and the operator can be constructed by a proper combination of causal and anti-causal filtering depending on the poles $\gamma_{i}$ of the system. The following proposition summarizes how to obtain the discrete counterpart $\Delta_{L}$ of $L$. The proof can be found in the Appendix.

Proposition 1 (Discrete Implementation of L): Consider the continuous-domain linear differential operator $L=L_{n} L_{d}^{-1}$, where $L_{n}$ and $L_{d}$ constitute the operators of $L$; the operator $L_{d}$ is inverted as before. Therefore,

$$
\begin{aligned}
L_{n} & =\prod_{i=1}^{N}\left(D-\alpha_{i} I\right) \\
L_{d} & =\prod_{i=1}^{M}\left(D-\gamma_{i} I\right) .
\end{aligned}
$$

We separate $L_{d}$ in its causal $\left(L_{d}^{\prime}\right.$, characterized by $\gamma^{\prime}=$ $\left.\left(\gamma_{1}^{\prime}, \ldots, \gamma_{m_{1}}^{\prime}\right), \operatorname{Re}\left(\gamma_{k}^{\prime}\right)<0\right)$ and anti-causal $\left(L_{d}^{\prime \prime}, \gamma^{\prime \prime}=\right.$ $\left.\left(\gamma_{1}^{\prime \prime}, \ldots, \gamma_{m_{2}}^{\prime \prime}\right), \operatorname{Re}\left(\gamma_{k}^{\prime \prime}\right)>0\right)$ parts to assure stability. Then, the discrete operator $\Delta_{L}$ associated to $L$ can be obtained by a cascade of filtering operations corresponding to

$$
\begin{aligned}
\mathbf{y}^{\prime}[n] & =\mathbf{x}[n]-\sum_{k=1}^{m_{1}} \mathbf{y}^{\prime}[n-k] \Delta_{L_{d}^{\prime}}[k], \\
\mathbf{y}^{\prime \prime}[n] & =\mathbf{y}^{\prime}\left[n+m_{2}\right] \Delta_{L_{d}^{\prime \prime}}\left[-m_{2}\right]-\sum_{k=-m_{2}}^{-1} \mathbf{y}^{\prime \prime}[n-k] \Delta_{L_{d}^{\prime \prime}}[k], \\
\mathbf{y}[n] & =\sum_{k \in \mathbb{Z}} \mathbf{y}^{\prime \prime}[n-k] \Delta_{L_{n}}[k]
\end{aligned}
$$

where $\mathbf{x}$ is the input, $\mathbf{y}^{\prime}$ is the output of the first recursive filtering step (causal), $\mathbf{y}^{\prime \prime}$ is the output of the second recursive

\footnotetext{
${ }^{1}$ For the remaining of the paper, we use $\Delta_{L}$ either as a convolution operator or as a discrete filter. The sense will be clear from the context.
} 


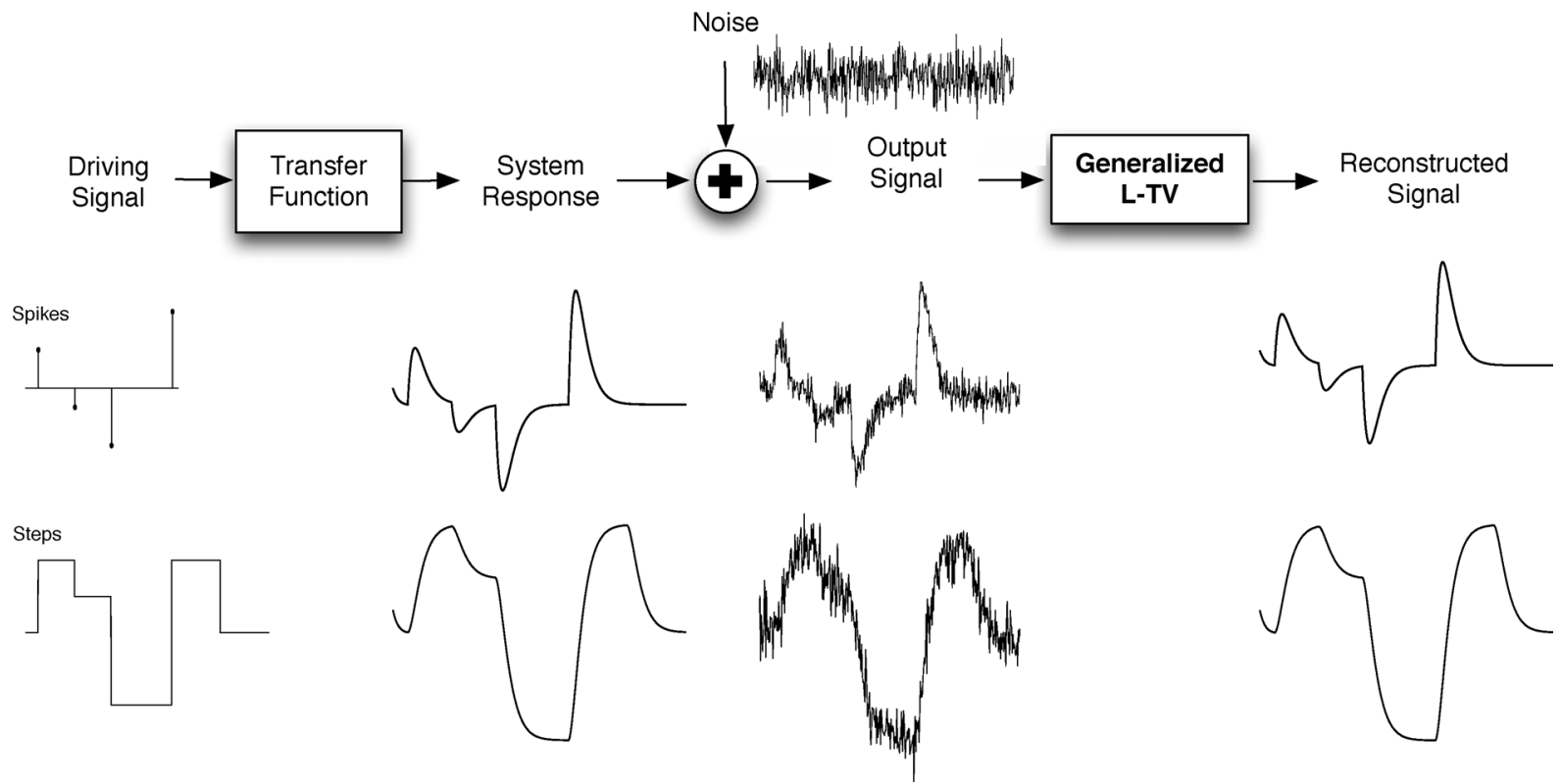

Fig. 1. Illustration showing the observation model and the use of generalized TV. First, the driving signal serves as input to the linear system. Next, the (ideal) system response gets corrupted by noise. Generalized TV regularization aims at minimizing the $\ell_{1}$-norm of the differential operator-which is tuned to the linear system and driving signal — applied to the reconstruction.

filtering step (anti-causal) and $\mathbf{y}$ is the final output. The constituting filters are given in the time domain by

$$
\begin{aligned}
& \Delta_{L_{n}}[n]=(-1)^{n} \sum_{|\mathbf{m}|=n}\left(e^{\boldsymbol{\alpha}}\right)^{\mathbf{m}}, \mathbf{m} \in[0,1]^{N}, 0 \leq n \leq N \\
& \Delta_{L_{d}^{\prime}}[n]=(-1)^{n} \sum_{|\mathbf{m}|=n}\left(e^{\boldsymbol{\gamma}^{\prime}}\right)^{\mathbf{m}}, \mathbf{m} \in[0,1]^{m_{1}}, 0 \leq n \leq m_{1} \\
& \Delta_{L_{d}^{\prime \prime}}[n]=(-1)^{n} \sum_{|\mathbf{m}|=-n}\left(e^{-\gamma^{\prime \prime}}\right)^{\mathbf{m}}, \mathbf{m} \in[0,1]^{m_{2}},-m_{2} \leq n \leq 0
\end{aligned}
$$

where we use the multi-index notation $\mathbf{m}=\left(m_{1}, \ldots, m_{N}\right)$, with $|\mathbf{m}|=\sum_{k=1}^{N} m_{k}$ and the conventions $c^{\mathbf{m}}=$ $\left(c^{m_{1}}, \ldots, c^{m_{N}}\right)$ and $\mathbf{c}^{\mathbf{m}}=\prod_{k=1}^{N} c_{k}^{m_{k}}$. The initial conditions for the recursive implementations (the last two rows of (7)) can be determined with respect to the input $\mathbf{x}$; i.e., $\mathbf{y}^{\prime}$ and $\mathbf{y}^{\prime \prime}$ are nonzero within the support of $\mathbf{x}$.

\section{B. TV Regularization}

As usual for TV regularization, we cast our problem of finding the approximation $\tilde{\mathbf{x}}$ from the noisy measurements $\mathbf{y}$ into a variation formulation where we use the generalized TV regularizer from (5). Then, the minimization problem becomes

$$
\tilde{\mathbf{x}}=\arg \min _{\mathbf{x}} \underbrace{\frac{1}{2}\|\mathbf{y}-\mathbf{x}\|_{2}^{2}+\lambda\left\|\Delta_{L}\{\mathbf{x}\}\right\|_{1}}_{J(\mathbf{x})}
$$

where $\lambda$ is the regularization tuning parameter and $\Delta_{L}$ is the discretized form of a differential operator of the form (6). The solution depends on $\lambda$, which controls the trade off between the data and regularization terms.

The optimal solution to (8) should find a compromise between data fidelity and regularization cost. In the continuous domain, any homogeneous solution $x_{0}(t)$ of the differential operator $L$ has no cost since $L\left\{x_{0}\right\}(t)=0$. This property also holds for the sampled version $\mathrm{x}_{0}$ and the discrete filter $\Delta_{L}$; i.e., $\Delta_{L}\left\{\mathbf{x}_{0}\right\}=0$. Therefore, null-space components of the differential operator can be used at no cost to minimize the residual error. Moreover, signals with a sparse representation after applying $\Delta_{L}$ have a low cost for the $\ell_{1}$-norm and will be preferred. For the case $L=D$, we retrieve the conventional TV regularization where the constant signal is the (only) null-space component and the lowest global cost is attained for piecewise-constant signals.

\section{On the Choice of Differential Operator $L$}

The main working principle of TV is to impose sparsity through the $\ell_{1}$-norm of the derivative of the signal, which is typically satisfied for piecewise-constant signals when combined with the data term. The additional flexibility that we propose by generalizing for any differential operator allows incorporating additional or different prior information.

When dealing with a linear system in Fig. 1, the differential operator can be tuned to the inverse of the system response. In this case, the differential operator will undo the effect of the linear system, and regularization will be guided by the driving signal. The $\ell_{1}$-norm leads to the optimal performance when the signal is spike-like. More complex driving signals can be dealt with by further refining the operator; e.g., for a step-like driving signal a regular derivative can be added to the regularizing operator. We will discuss this point in more detail in Section IV.

\section{OPTIMIZATION ALGORITHMS}

In what follows, we briefly review two practical algorithms to obtain the minimizer of the cost functional $J(\mathbf{x})$ in (8). For now, we assume that all vectors and matrices involved are finite-dimensional. This minimization problem can be regarded as a generalized form of the analysis-prior denoising problem (see [6] and [26] and the references therein). The first algorithm is essentially an adaptation of the one provided by Chambolle for TV denoising [5], [31] (also see [19] for a slightly different algorithm). In [5], the (fast) gradient projection ((F)GP) algorithm is derived for TV denoising problems $\left(\Delta_{L}=\Delta\right)$ whereas we adapted the algorithm for the discrete operator $\Delta_{L}$. 
Algorithm 1: Let us consider the regularization operator $\Delta_{L}$; its $z$-transform is given by $\widehat{\Delta}_{L}(z):=\sum_{n} \Delta_{L}[n] z^{-n}$ and $z=$ $e^{j \omega}$ reverts to the discrete time Fourier transform of $\Delta_{L}[n]$. Further more, we choose $c$ subject to

$$
c>\sup _{\omega}\left|\widehat{\Delta}_{L}\left(e^{j \omega}\right)\right|^{2}=\sup _{\omega} \frac{\prod_{i=1}^{N}\left|1-e^{\alpha_{i}} e^{-j \omega}\right|^{2}}{\prod_{i=1}^{M}\left|1-e^{\gamma_{i}} e^{-j \omega}\right|^{2}} .
$$

The algorithm then iterates the following steps until convergence:

I) Update $\mathbf{z}:=\Delta_{L}\{\mathbf{y}\} /(\lambda c)+\left(I-\Delta_{L} \Delta_{L}^{T} / c\right)\{\mathbf{z}\}$, where the adjoint of $\Delta_{L}$ is $\Delta_{L}^{T}[n]=\Delta_{L}^{*}[-n]$.

II) Update $\mathbf{z}:=\operatorname{clip}(\mathbf{z})$ where $\operatorname{clip}(\cdot)$ denotes the element-wise clipping function,

$$
\operatorname{clip}(\cdot)=\operatorname{sign}(\cdot) \min (|\cdot|, 1) .
$$

After convergence, set $\mathbf{x}=\mathbf{y}-\lambda \Delta_{L}^{T}\{\mathbf{z}\}$.

With a small variation only, the fast version of the algorithm can be obtained [5].

Algorithm 2: Repeat the following steps until the convergence criterion is met for each $i^{\text {th }}$ iteration with $k_{1}=1$ :

I) Update $\mathbf{z}_{i}:=\Delta_{L}\{\mathbf{y}\} /(\lambda c)+\left(I-\Delta_{L} \Delta_{L}^{T} / c\right)\left\{\mathbf{v}_{i}\right\}$.

II) Update $\mathbf{z}_{i}:=\operatorname{clip}\left(\mathbf{z}_{i}\right)$.

III) Update $k_{i+1}=\frac{1+\sqrt{1+4 k_{i}^{2}}}{2}$.

IV) Update $\mathbf{v}_{i+1}=\mathbf{z}_{i}+\frac{k_{i}-1}{k_{i+1}}\left(\mathbf{z}_{i}-\mathbf{z}_{i-1}\right)$.

After convergence, set $\mathbf{x}=\mathbf{y}-\lambda \Delta_{L}^{T}\{\mathbf{z}\}$.

These algorithms make use of the dual-problem [19] and employs the "fast gradient-projection" (FGP) method [5]. Equivalent algorithms can be derived employing the "majorizationminimization" scheme (see [32]-[34]). Again initiating from the dual form, Chambolle [19] proposes a slightly different gradient-based model; finally, similar algorithms [23], [31] suggest "graph-cut" technique to solve the same problem. We refer to [35] for an extensive overview of recent algorithms.

It is important to note that generalized TV can be combined with the more general deconvolution problem: the data term may include a matrix/operator $\mathbf{A}$; e.g., that corresponds to a blurring filter. Then the optimization problem turns into

$$
\tilde{\mathbf{x}}=\arg \min _{\mathbf{x}} \frac{1}{2}\|\mathbf{y}-\mathbf{A x}\|_{2}^{2}+\lambda\left\|\Delta_{L}\{\mathbf{x}\}\right\|_{1} .
$$

The solution of this problem is not very practical to achieve directly with Algorithms 1 and 2 since the inverse of $\mathbf{A}^{\mathbf{T}} \mathbf{A}$ may be ill-conditioned. The trick is to apply a two step optimization. The first (outer) loop tackles the deblurring problem while the second (inner) loop solves the denoising problem as proposed here. For more details, we refer to [5 Sec. 5] for combining (F)ISTA/FGP algorithms, and to [36] for the "majorization-minimization" algorithm.

\section{EXPERIMENTAL RESULTS}

In this section, we present several examples to illustrate the usefulness and performance of the proposed method. First, we demonstrate signal reconstruction of a simulated linear system response with different driving signals and show how it outperforms conventional TV. Finally, we show waveform analysis of audio signals by tuning the zeros of the operator to the central tone.
TABLE I

The Differential Operator $L$ FOR Generalized TV NeEds to Be CHOSEN ACCording to the Linear System AND the TyPe OF Driving SigNAL. $I$ IS THE IDENTITY OPERATOR. THE INVERSE IS DEFINED AS EXPLAINED IN SECTION IV-A

\begin{tabular}{l|c|c}
\hline \hline driving signal & linear system & optimal TV operator $L$ \\
\hline \hline spikes & $I$ & $I$ \\
piecewise constant & $I$ & $D$ (conventional TV) \\
spikes & $\tilde{h}=\tilde{L}^{-1}\{\delta\}$ & $\tilde{L}$ \\
piecewise constant & $\tilde{h}=\tilde{L}^{-1}\{\delta\}$ & $D \tilde{L}$ \\
piecewise linear & $\tilde{h}=\tilde{L}^{-1}\{\delta\}$ & $D^{2} \tilde{L}$ \\
\hline \hline
\end{tabular}

\section{A. Proof of Concept}

We present synthetic examples according to the model shown in Fig. 1 to demonstrate the usefulness of generalized TV regularization. To focus the attention, we consider a linear system

$$
\tilde{h} * s=x
$$

where $s$ and $x$ are the input and output signals, respectively, and * denotes the convolution operator. We introduce the continuous Fourier transform of $\tilde{h}(t)$ as $\widehat{\tilde{h}}(\omega):=\int_{t} \tilde{h}(t) e^{-j \omega t} d t$.

We can define a pseudoinverse $\tilde{L}\{x\}=\tilde{g} * x$, where $\tilde{g}$ is defined by

$$
\hat{\tilde{g}}(\omega)= \begin{cases}1 / \hat{\tilde{h}}(\omega), & \text { if } \hat{\tilde{h}}(\omega) \neq 0 \\ \hat{\tilde{h}}(\omega)=0, & \text { if } \hat{\tilde{h}}(\omega)=0 .\end{cases}
$$

Then, matching the regularization operator $L$ with $\tilde{L}$ will cancel the effect of the linear system:

$$
L\{x\}=L\{\tilde{h} * s\}=\tilde{g} * \tilde{h} * s=s+s_{\text {null }}
$$

where $s_{\text {null }}$ is a null-space component of the system $\tilde{h}$, in other words, $\tilde{h} * s_{\text {null }}=0$. Notice that a null-space component of $L$ cannot be recovered.

Depending on the assumptions on the driving signal $s$, we have the additional freedom to include derivative operators in $L$ in order to further sparsify the signal and make the $\ell_{1}$-norm effective. Specifically, in Table I, we give an overview of how the operator $L$ should be chosen for various types of driving signals; e.g., spikes, piecewise-constant, and piecewise-linear.

We now illustrate these concepts by considering a third-order system driven by a spike-like signal. In this case, the Fourier domain of the impulse response of the system has the form

$$
\widehat{\tilde{h}}(\omega)=\frac{\left(j \omega-\tilde{\gamma}_{1}\right)}{\left(j \omega-\tilde{\alpha}_{1}\right)\left(j \omega-\tilde{\alpha}_{2}\right)\left(j \omega-\tilde{\alpha}_{3}\right)}
$$

with three poles and one zero. Consequently, the differential operator $\tilde{L}$ can be characterized in its turn in the Fourier domain as the inverse of $\tilde{h}$ :

$$
\widehat{\tilde{L}}(\omega)=\frac{\left(j \omega-\tilde{\alpha}_{1}\right)\left(j \omega-\tilde{\alpha}_{2}\right)\left(j \omega-\tilde{\alpha}_{3}\right)}{\left(j \omega-\tilde{\gamma}_{1}\right)}
$$

where the system's poles take the role of the operator's zeros (and vice versa). The time-domain operator then corresponds to

$$
\tilde{L}=\left(D-\tilde{\alpha}_{1} I\right)\left(D-\tilde{\alpha}_{2} I\right)\left(D-\tilde{\alpha}_{3} I\right)\left(D-\tilde{\gamma}_{3} I\right)^{-1} .
$$

For a practical example, we take $\tilde{\alpha}=\tilde{\alpha}_{1}=\tilde{\alpha}_{2}=\tilde{\alpha}_{3}=-2$ and $\gamma=-0.1$. 


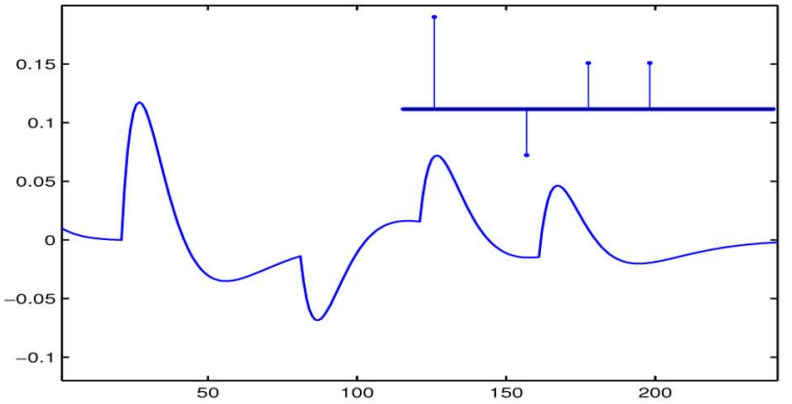

(a)

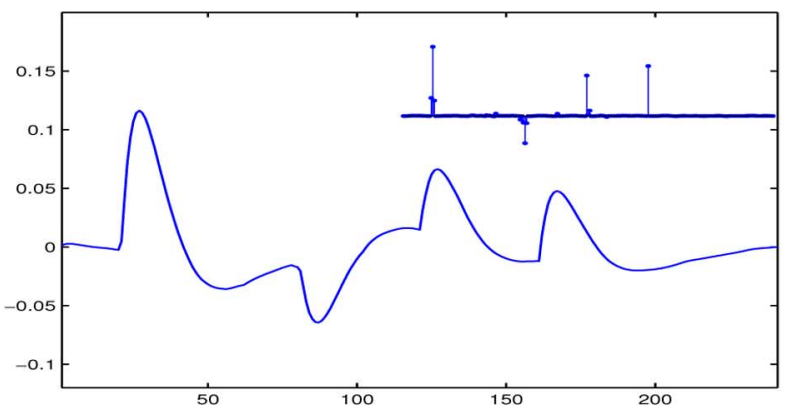

(c)

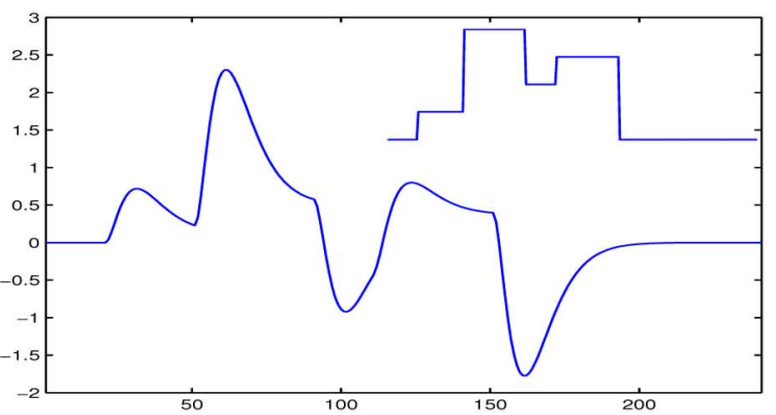

(e)

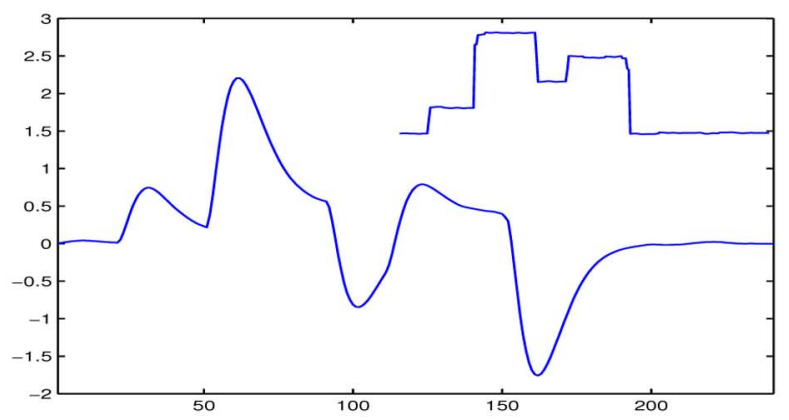

(g)

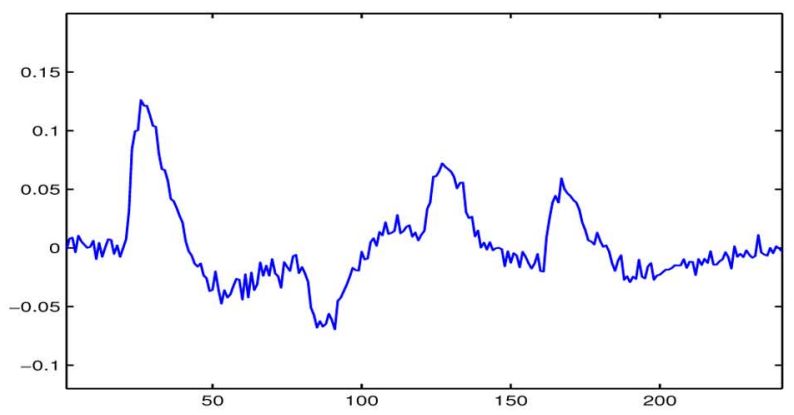

(b)

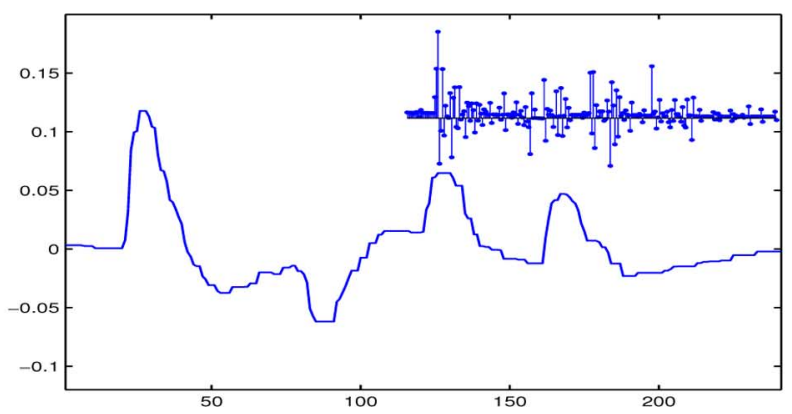

(d)

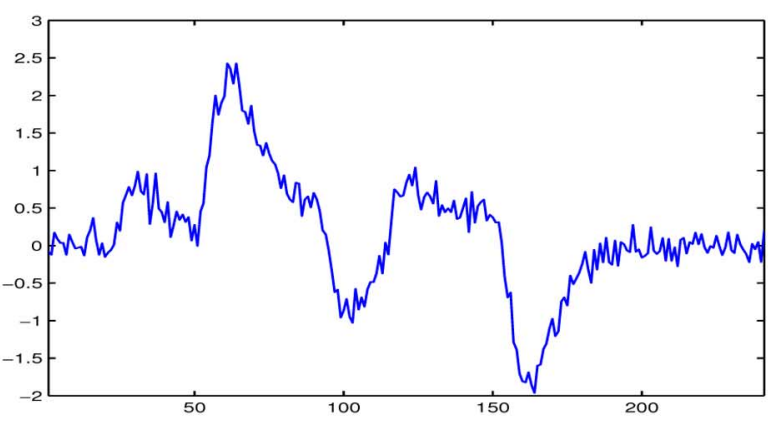

(f)

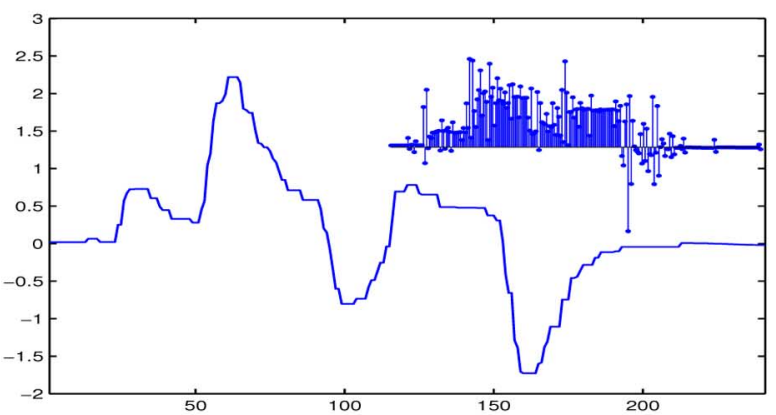

(h)

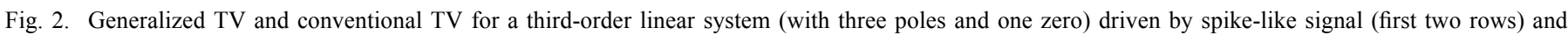

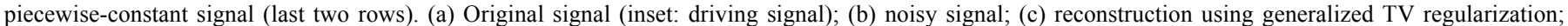

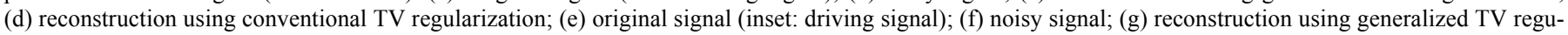
larization; (h) reconstruction using conventional TV regularization.

Since we assume the driving signal to be spike-like, we select $L=\tilde{L}$, for which the discrete version $\Delta_{L_{n}}$ of the forward operator corresponds to an FIR filter with four taps: $\left[1,-3 e^{\alpha}, 3 e^{2 \alpha},-e^{3 \alpha}\right]$ and the discrete version of the inverse op- 
TABLE II

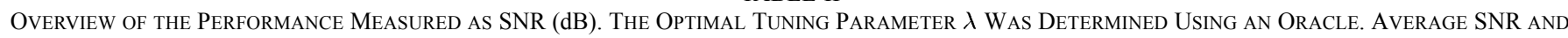
ITS STANDARD DEVIATION ARE REPORTED FOR 100 REALIZATIONS OF THE NOISE

\begin{tabular}{|c|c|c|c|c|c|c|}
\hline & SNR (dB) & $\lambda$ & SNR (dB) & $\lambda$ & SNR (dB) & $\lambda$ \\
\hline noisy signal 1 & 5 & - & 10 & - & 20 & - \\
\hline e Wiener & $14.09 \pm 0.87$ & - & $17.45 \pm 0.65$ & - & $24.31 \pm 0.41$ & - \\
\hline entional TV & $12.32 \pm 0.68$ & $0.32 \pm 0.04$ & $15.89 \pm 0.63$ & $0.16 \pm 0.05$ & $22.15 \pm 0.46$ & $0.10 \pm 0.01$ \\
\hline generalized TV & $14.86 \pm 1.96$ & $0.82 \pm 0.38$ & $19.58 \pm 2.22$ & $0.52 \pm 0.25$ & $28.71 \pm 1.92$ & $0.17 \pm 0.09$ \\
\hline noisy signal 2 & 5 & - & 10 & - & 20 & - \\
\hline iener & $14.17 \pm 0.88$ & - & $18.87 \pm 0.84$ & - & $26.90 \pm 0.59$ & - \\
\hline entional TV & $13.08 \pm 0.72$ & $1.96 \pm 0.36$ & $16.70 \pm 0.64$ & $0.98 \pm 0.17$ & $23.99 \pm 0.41$ & $0.22 \pm 0.05$ \\
\hline generalized TV & $14.83 \pm 1.68$ & $10.02 \pm 1.86$ & $19.78 \pm 1.86$ & $4.90 \pm 1.49$ & $29.35 \pm 1.60$ & $1.70 \pm 0.63$ \\
\hline
\end{tabular}

erator corresponds to a causal IIR filtering with $\Delta_{L^{\prime}}=\left[1,-e^{\gamma}\right]$. In Fig. 2(a), we show the noiseless original signal, the spike-like driving signal in the inset. We also added a random null-space component of the operator $\tilde{L}$ (i.e., $c_{1} e^{\tilde{\alpha} t}+c_{2} t e^{\tilde{\alpha} t}+c_{3} t^{2} e^{\tilde{\alpha} t}$ ) as a "background" to which the regularization term is insensitive. Next, the signal is corrupted by i.i.d. Gaussian noise 15 $\mathrm{dB}$ [see Fig. 2(b)] and approximated using two regularization methods: generalized TV and conventional TV as shown in Fig. 2(c) and (d), respectively. For each method the oracle selected the optimal regularization parameter $\lambda$ since it has access to the ground truth. We also compute the filtered version $\Delta_{L}$ of the regularized solution in order to explore how well we reconstruct the underlying driving signal, see the insets in Fig. 2(c) and (d). The choice of the regularization parameter $\lambda$ is important since the oracle is not available in practice. Different strategies can be adapted for selecting $\lambda$ (see [37] for classical references to methods such as generalized cross-validation and the L-curve, or [38] for a recent Monte Carlo adaptation of Stein's unbiased risk estimate that works well for TV, and [19], [39]-[42]).

As an additional experiment, we change the driving signal into piecewise-constant, as shown in Fig. 2. Accordingly, we adapt the regularizing operator into $L=D \tilde{L}$, which only leads to an alteration of the FIR filter, $\Delta_{L_{n}}=\left[1,-1-3 e^{\alpha}, 3 e^{2 \alpha}+\right.$ $\left.3 e^{\alpha}, 3 e^{\alpha}-e^{3 \alpha}, e^{3 \alpha}\right]$. The results for the generalized TV and conventional TV are shown in Fig. 2(g) and (h), respectively.

The regularization in (8) is performed using the algorithm described in Section III. We deploy three different noise settings: additive Gaussian noise corresponding to SNR level of 5, 10, and $20 \mathrm{~dB}$. We report average SNR levels with standard deviation (over 100 realizations), with the optimal regularization parameter and maximum SNR, in Table II. We compare the reconstruction quality obtained by oracle-Wiener filtering (optimal for a Gaussian-process driving signal corrupted with additive Gaussian noise), conventional TV, and generalized TV. As expected, the results reveal that generalized TV is superior to conventional TV and the Wiener filter when tuning the operator $L$ different from $D$ is appropriate. Moreover, as it can be appreciated from the corresponding Figures, also the reconstruction of the underlying driving signal has high quality and can be useful for further processing in applications.

An important concern is the robustness of the choice of the regularization operator $L$ with respect to the underlying "true" linear system. To that aim, we generate signals for a spike-like input of a third-order linear system $\tilde{L}=(D-\tilde{\alpha} I)^{3}(D-\gamma I)^{-1}$ with $\tilde{\alpha}$ in the range $[-2,2]$. We create 10 different realizations corrupted by additive Gaussian noise resulting into $10 \mathrm{~dB}$ SNR. Next, we apply several regularization strategies: 1) generalized TV with $\alpha$ tuned exactly to the system;2) generalized

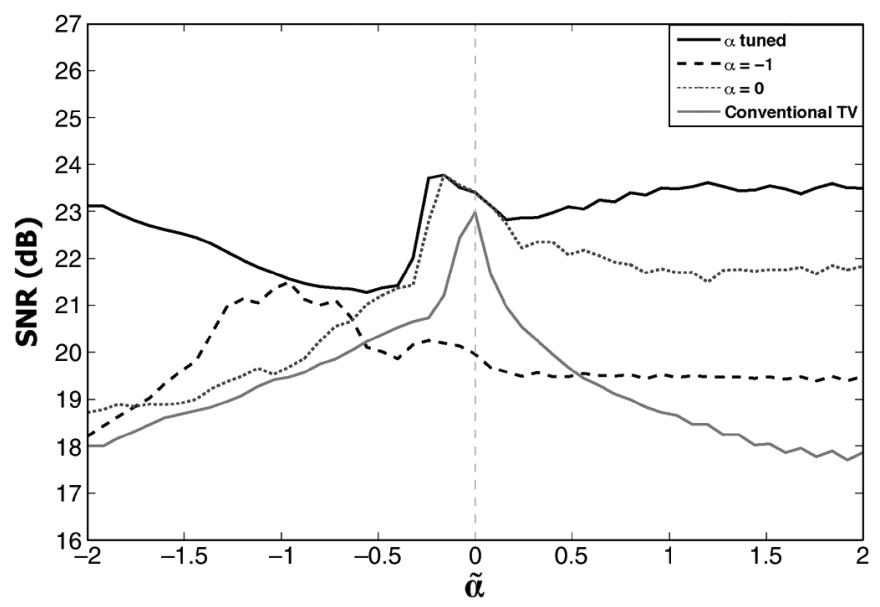

Fig. 3. Performance measured as SNR (dB) for generalized TV and conventional TV regularization of a third-order linear system with equivalent differential operator $\tilde{L}=(D-\tilde{\alpha} I)^{3}(D-\gamma I)^{-1}$, with $\gamma=4$, for varying $\tilde{\alpha}$ values. The reported SNR measures are averaged over 10 realizations of additive Gaussian noise (noisy signal $10 \mathrm{~dB}$ ).

TV with $\alpha=-1$;3) generalized TV with $\alpha=0$ to illustrate second-order TV; and 4) conventional TV. We kept $\gamma=4$ constant to eliminate pole-zero cancellation. In Fig. 3, we plot the average SNR (10 regularizations) for different $\tilde{\alpha}$ values of the linear system. As expected, we observe that second-order TV and matched generalized TV have equal performance at $\tilde{\alpha}=0$. Similarly, generalized TV with fixed $\alpha=-1$ meets matched generalized TV at $\tilde{\alpha}=-1$. Moreover, we notice that SNR levels for matched generalized TV tend to increase further for larger values of $\tilde{\alpha}$. The Green's function of the differential operator $\tilde{L}$ increases which generates high correlation between the samples. Conventional TV underperforms as the derivative operator is not well tuned to the linear system.

\section{B. Sound Waveform Processing}

We show that it is possible to tune the operator of generalized TV to include information about modulation, which can be useful for audio signals; e.g., processing tonal and transients layers [43].

Specifically, let us assume the simplified signal model as a sum of shifted decaying exponentials, each one modulated by a high-frequency sinusoidal function:

$$
y(t)=\sum_{i} A_{i} \sin \left(\omega_{0, i}\left(t-t_{i}\right)\right) e^{\alpha\left(t-t_{i}\right)} u\left(t-t_{i}\right)
$$

where $\alpha$ is the decay rate. (10) can be considered as the sum of responses of linear systems with impulse responses $\sin \left(\omega_{0, i} t\right) e^{\alpha t} u(t)$ for spikes $A_{i} \delta\left(t-t_{i}\right)$, respectively. Here we 


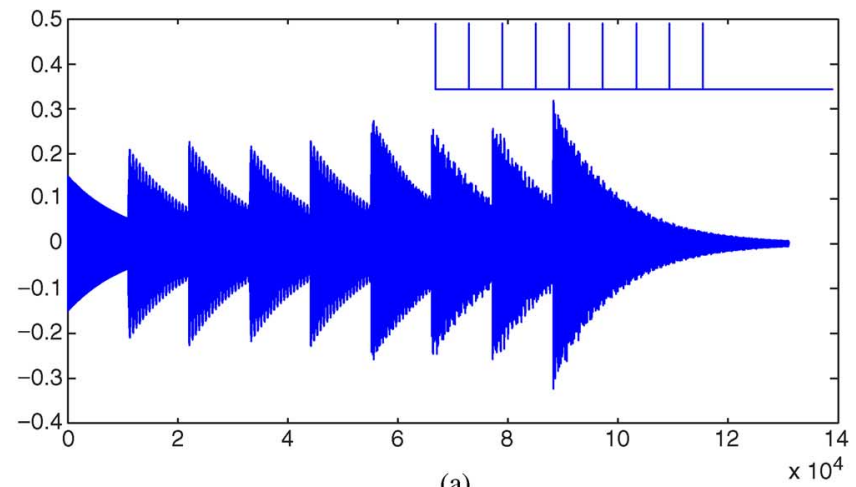

(a)

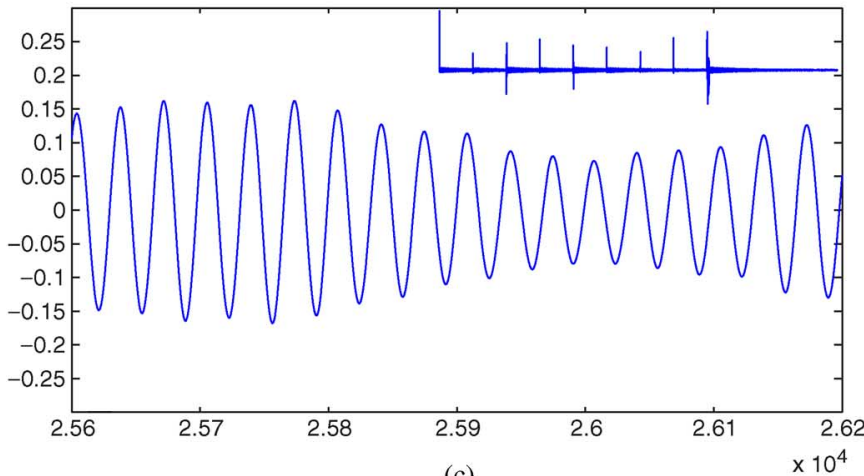

(c)

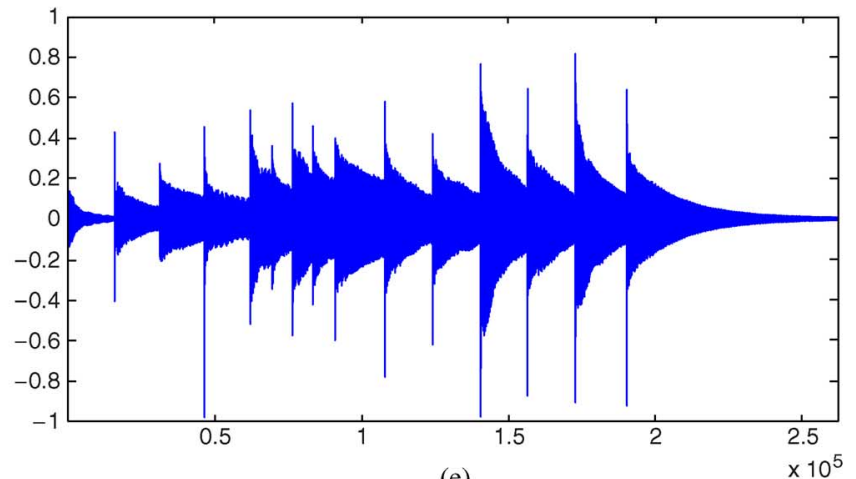

(e)

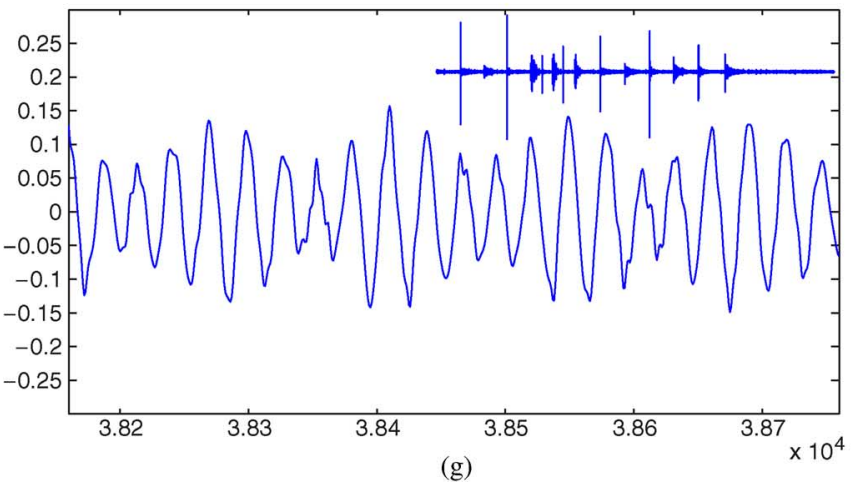

(g)

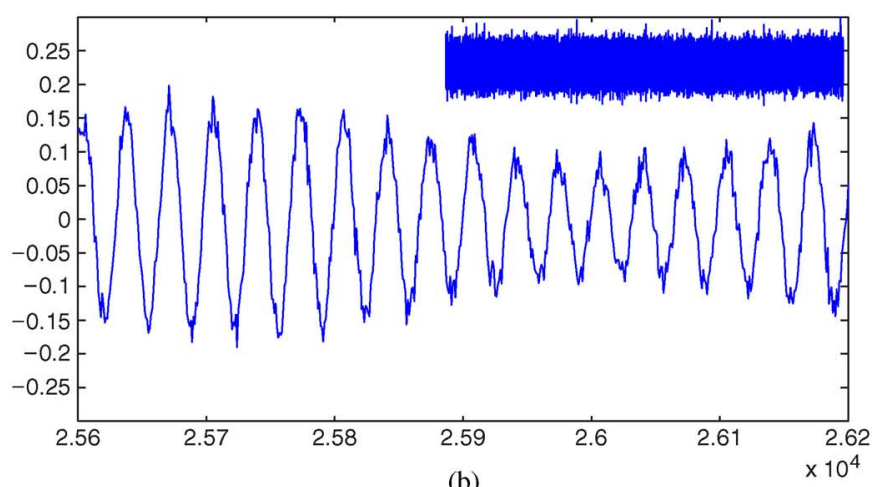

(b)

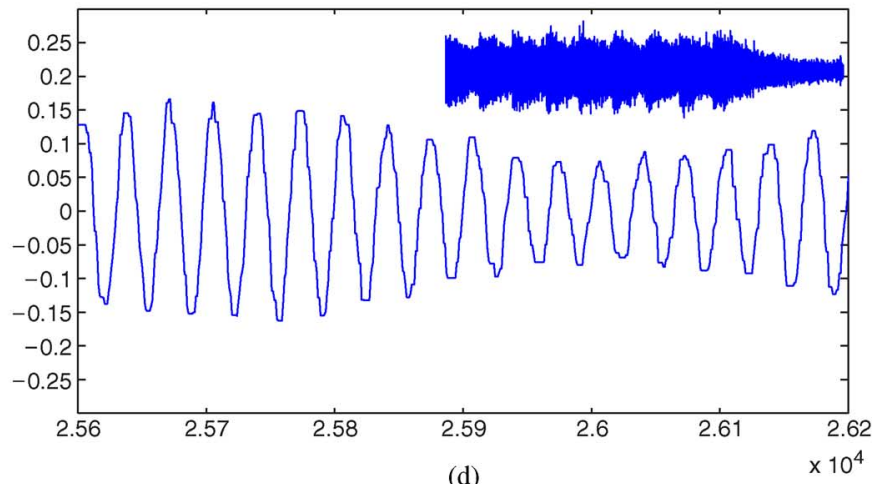

(d)

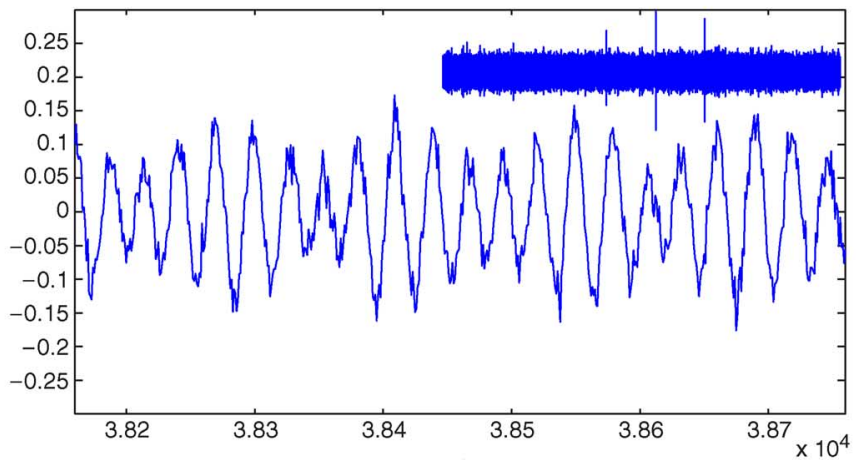

(f)

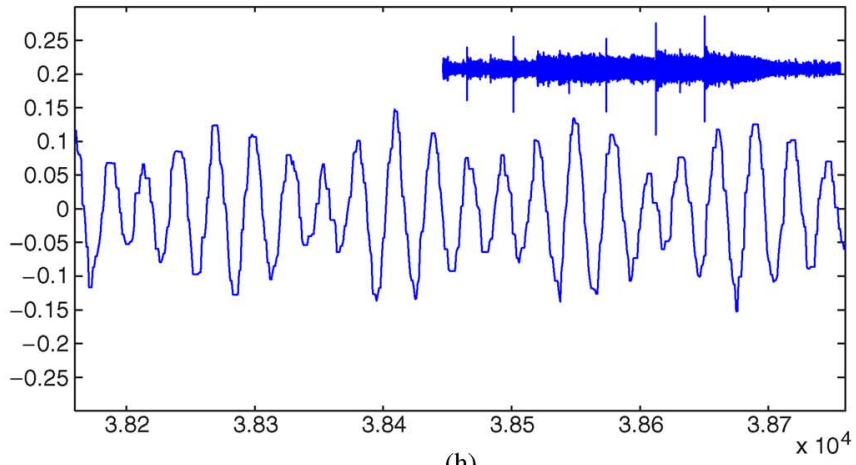

(h)

Fig. 4. Zoom of the denoised audio signal, Für Elise (first two rows) and Glockenspiel signal (last two rows) corrupted with i.i.d. $\backslash$ Gaussian noise $15 \mathrm{~dB}$ with generalized TV and conventional TV. We employ differential operator $L=\left(D+\left(j \omega_{0}+\alpha\right) I\right)\left(D+\left(-j \omega_{0}+\alpha\right) I\right)$ and $L=\left(\left(D+\left(j \omega_{0}+\alpha\right) I\right)(D+\right.$ $\left.\left.\left(-j \omega_{0}+\alpha\right) I\right)\right)^{3}$ for Für Elise and Glockenspiel signal, respectively, where $\omega_{0}$ is the average frequency and $\alpha=-4 \mathrm{~s}^{-1}$. (a) Original signal; (b) noisy signal; (c) reconstruction using generalized TV regularization; (d) reconstruction using conventional TV regularization; (e) original glockenspiel signal; (f) noisy signal; (g) reconstruction using generalized TV regularization; and (h) reconstruction using conventional TV regularization.

pick one (central) frequency $\omega_{0}$, for which the corresponding transfer function is

$$
\widehat{\tilde{h}}(w)=\frac{\omega_{0}}{\left(j \omega+\left(j \omega_{0}+\alpha\right)\right)\left(j \omega+\left(-j \omega_{0}+\alpha\right)\right)}
$$

and the associated second-order differential operator becomes $L=\left(D+\left(j \omega_{0}+\alpha\right) I\right)\left(D+\left(-j \omega_{0}+\alpha\right) I\right)$.

We generated a synthetic signal [Fig. 4(a)] according to (10) with the first 9 notes of Für Elise whose frequencies range from $329-1318 \mathrm{~Hz}$ and at sampling frequency $f_{s}=44100 \mathrm{~Hz}$. The 
decay rate was $\alpha=-4 \mathrm{~s}^{-1}$. We used the average frequency of the notes and $\alpha=-4 \mathrm{~s}^{-1}$. The effect of tuning $\alpha$ is negligible on the output since the decay is very slow compared to the sampling frequency. We created a noisy realization of the audio signal by corrupting it with i.i.d. Gaussian noise at SNR $=15 \mathrm{~dB}$, see Fig. 4(b). We show the output of generalized $(25.3 \mathrm{~dB})$ and conventional TV $(18.3 \mathrm{~dB})$ in $4(\mathrm{c})$ and (d), respectively. The regularization parameter $\lambda$ was chosen using an oracle.

In the supplementary material, we have provided audio files of the example given above; i.e., original, noisy, denoised using conventional and generalized TV, and the spike driving signal can be listened. We also include another example using the real-world test signal "Glockenspiel" [43], where we increase the multiplicity of the zeros to make the frequency response of $L$ increasingly flat around $\omega_{0}$, and thus cancel also tones with nearby frequencies. ${ }^{2}$ Finally, in Fig. 4(e), we show the "Glockenspiel" signal, which is directly adopted from [43]. We degraded the signal with additive i.i.d. Gaussian noise at SNR = $15 \mathrm{~dB}$; see Fig. 4(f). The output for generalized TV (18.3 dB) and conventional TV $(16.67 \mathrm{~dB})$ is shown in $4(\mathrm{~g})$ and $(\mathrm{h})$, respectively. Note that the SNR values are computed against the real "Glockenspiel" signal, which contains some noise itself and consequently the comparison with the original signal is not ideal. First, the (noiseless) ground truth is not known. Second, the signal contains different frequency components from perturbed harmonics in the tonal layer and sharp transitions in the transient layer, neither of which are modeled by the operator in generalized TV - nevertheless, the result is still better than for conventional TV. More advanced applications of generalized TV for sound wave processing can be devised in future, such as the inclusion of multiple regularization terms with different operators each (e.g., for different frequencies and harmonics) and an additional model to deal with the transient layer.

\section{CONCLUSION}

TV regularization has become a widely applied scheme with edge-preserving properties. Extensions have mainly focused on the use of higher order derivatives [12]-[17] and recently also on nonlocal generalizations [44]. In this paper, we extended the basic TV concept further by introducing a general differential operator $L$ instead of the derivative $D$. This allows a great deal of flexibility since we can take into account the presence of a linear system and different types of driving signals. From digital signal processing view, we build the simplest discrete approximation of the differential operator $L$ and we define them explicitly as FIR/IIR filters. Generalized TV scheme is appealing for many signal processing applications, in particular, when the system can be expressed or approximated in terms of differential operators. We illustrated our framework by considering a third-order linear system. In order to solve the regularization problem, we proposed an alternative algorithm based on the dual form of TV. This work can be considered as the analysis prior counterpart of exponential spline wavelets [27] or generalized Daubechies wavelets [28]; indeed, these wavelets are generalizations that can be tuned to a given differential operator and their use in regularized reconstruction

\footnotetext{
${ }^{2}$ Increasing the multiplicity of the zeros increases the size of the nullspace. I is also equivalent to the number of vanishing moments of a wavelet transform.
}

concurs with a synthesis prior. Despite the fact that our approach is heavily inspired on the continuous domain, future research is needed to tighten the mathematical link between the proposed signal processing approach (in the discrete domain) and proper generalization of TV in the continuous domain. We believe that our approach is promising in this respect because in recent work [45] it was shown that the signal-processing approach for conventional TV (that is, $\ell_{1}$-norm of finite differences) can be linked to proper continuous-domain modeling of stochastic processes.

Finally, we mention that another application of generalized TV could be to perform system identification; i.e., when strong assumptions on the (sparsity of the) driving signal can be made, one can attempt to retrieve the form of $L$ and, for model selection, evaluate the performance of the estimated operator by statistical methods such as Akaike's information criterion and its extension for the state-space representation of linear systems [46].

\section{APPENDIX}

\section{PROOF OF PROPOSITION 1}

Proof: We make the proof by construction. For the firstorder differential operator $L=\left(D-\alpha_{1}\right)$ for which $N=1$ and $M=0$, the corresponding discrete operator, $\Delta_{L}$, becomes $\Delta_{L}\{\mathbf{x}\}[n]=\mathbf{x}[n]-e^{\alpha_{1}} \mathbf{x}[n-1]$; see [47].

For the differential operator $L=\prod_{i=1}^{N}\left(D-\alpha_{i} I\right)$ of order $N>0$ and $M=0$, we can obtain the filter $\Delta_{L}$ by successive convolutions (leading to support of $N+1$ ); the $z$-transform of $\widehat{\Delta}_{L}(z)$ is then

$$
\widehat{\Delta}_{L}(z):=\sum_{n=0}^{N} \Delta_{L}[n] z^{-n}=\prod_{i=1}^{N}\left(1-e^{\alpha_{i}} z^{-1}\right)=\prod_{i=1}^{N} p_{i}\left(z^{-1}\right)
$$

where $p_{i}$ is a polynomial with 2 coefficients $p_{i, k}=\left(-e^{\alpha_{i}}\right)^{k}$ with $k \in[0,1]$. Note that we can express (12) benefiting the polynomial multiplication which leads to the convolution as

$$
\prod_{i=1}^{N} p_{i}\left(z^{-1}\right)=\sum_{n=0}^{N} \mathbf{P}_{N}[n] z^{-n}
$$

where $\mathbf{P}_{N}=p_{1} * p_{2} * \ldots * p_{N}$ and $*$ is the convolution operator. Therefore, we can express the filter $\Delta_{L}[n]=\mathbf{P}_{N}[n]$ as

$$
\begin{aligned}
\mathbf{P}_{N}[n]= & \sum_{k_{N-1}=0}^{n} \ldots \sum_{k_{2}=0}^{k_{3}} \sum_{k_{1}=0}^{k_{2}}\left(-e^{\alpha_{1}}\right)^{k_{1}}\left(-e^{\alpha_{2}}\right)^{k_{2}-k_{1}} \\
& \ldots\left(e^{\alpha_{N}}\right)^{n-k_{N-1}}, \quad\left\{k_{1}, \ldots, n-k_{N-1}\right\} \in[0,1]^{N}
\end{aligned}
$$

with a change of variables $m_{1}=k_{1}, m_{i}=k_{i}-k_{i-1}, m_{N}=$ $n-k_{N-1}$ we have

$$
\Delta_{L}[n]=(-1)^{n} \sum_{|\mathbf{m}|=n}\left(e^{\boldsymbol{\alpha}}\right)^{\mathbf{m}}, \quad \mathbf{m} \in[0,1]^{N}, 0 \leq n \leq N .
$$

Similarly, for the general differential operator, $L$, with $M>0$ we have in $z$-domain

$$
\widehat{\Delta}_{L}(z)=\widehat{\Delta}_{L_{n}}(z) \widehat{\Delta}_{L_{d}}^{-1}(z)=\frac{\prod_{i=1}^{N}\left(1-e^{\alpha_{i}} z^{-1}\right)}{\prod_{i=1}^{M}\left(1-e^{\gamma_{i}} z^{-1}\right)}
$$

where $\Delta_{L_{n}}[n]$ is represented explicitly in (14). Note that the filter $\widehat{\Delta}_{L_{d}}^{-1}$ in (15) has infinite support in time, therefore stability should be assured. Depending on the poles $\gamma$ of the operator, we 
find a combination of causal $\left(\boldsymbol{\gamma}^{\prime}\right)$ and anti-causal $\left(\boldsymbol{\gamma}^{\prime \prime}\right)$ filters that guarantee stability; e.g., for $M=1$ and $N=0$, we have either $\Delta_{L}[n]=e^{\gamma^{\prime} n} u[n]$ or $\Delta_{L}[n]=-e^{\gamma^{\prime \prime} n} u[-n-1]$, where $u[n]$ is the unit step function. In practice, the filter with input $\mathbf{x}$ and output $\mathbf{y}$ can be reformulated and implemented as in a recursive way by providing the realization of causal and anti-causal parts separately. To this aim, we represent the inverse filter

$$
\widehat{\Delta}_{L_{d}}^{-1}(z)=\widehat{\Delta}_{L_{d}^{\prime}}^{-1}(z) \widehat{\Delta}_{L_{d}^{\prime \prime}}^{-1}(z) \mathbf{e}^{-\gamma^{\prime \prime}}(-z)^{m_{2}}
$$

by the causal and anti-causal filters

$$
\begin{aligned}
& \widehat{\Delta}_{L_{d}^{\prime}}(z)=\prod_{i=1}^{m_{1}}\left(1-e^{\gamma_{i}^{\prime}} z^{-1}\right) \\
& \widehat{\Delta}_{L_{d}^{\prime \prime}}(z)=\prod_{i=1}^{m_{2}}\left(1-e^{-\gamma_{i}^{\prime \prime} z}\right)
\end{aligned}
$$

where $\mathbf{e}=(e, \ldots, e)$ is a vector of length $m_{2}$. Then the corresponding recursive algorithm can be easily derived from the $z$-domain representation. Here we will concentrate on the anticausal part (the derivation for the causal part is similar). To obtain $\mathbf{y}=\Delta_{L_{d}^{\prime \prime}}^{-1}\left\{\mathbf{x}\left[n+m_{2}\right](-1)^{m_{2}} \mathbf{e}^{-\gamma^{\prime \prime}}\right\}$, we consider

$$
\widehat{\mathbf{y}}(z) \widehat{\Delta}_{L_{d}^{\prime \prime}}(z)=\mathbf{e}^{-\gamma^{\prime \prime}}(-z)^{m_{2}} \widehat{\mathbf{x}}(z)
$$

from which we find

$$
\sum_{k} \mathbf{y}[n-k] \Delta_{L_{d}^{\prime \prime}}[k]=\mathbf{x}\left[n+m_{2}\right](-1)^{m_{2}} \mathbf{e}^{-\gamma^{\prime \prime}}
$$

From (14), we can derive the explicit time domain expression for the anti-causal filter $\Delta_{L_{d}^{\prime \prime}}$ as

$$
\Delta_{L_{d}^{\prime \prime}}[n]=(-1)^{n} \sum_{|\mathbf{m}|=-n}\left(e^{-\gamma^{\prime \prime}}\right)^{\mathbf{m}}, \mathbf{m} \in[0,1]^{m_{2}},-m_{2} \leq n \leq 0 .
$$

Therefore, we obtain

$$
\mathbf{y}[n]=\mathbf{x}\left[n+m_{2}\right] \Delta_{L_{d}^{\prime \prime}}\left[-m_{2}\right]-\sum_{k=-m_{2}}^{-1} \mathbf{y}[n-k] \Delta_{L_{d}^{\prime \prime}}[k]
$$

where we used $\Delta_{L_{d}^{\prime \prime}}[0]=1$.

Let us give an example for the third-order differential operator $L=\prod_{i=1}^{3}\left(D-\alpha_{i} I\right)$. The FIR filter $\Delta_{L}[n]$ then becomes

$$
\begin{aligned}
\Delta_{L}[n]=\left[1,-\left(e^{\alpha_{1}}+e^{\alpha_{2}}+e^{\alpha_{3}}\right), e^{\left(\alpha_{1}+\alpha_{2}\right)}+e^{\left(\alpha_{1}+\alpha_{3}\right)}\right. & \\
& \left.+e^{\left(\alpha_{2}+\alpha_{3}\right)},-e^{\left(\alpha_{1}+\alpha_{2}+\alpha_{3}\right)}\right], \quad 0 \leq n \leq 3 .
\end{aligned}
$$

\section{ACKNOWLEDGMENT}

İ. Bayram would like to thank I. W. Selesnick, Polytechnic Institute of New York University, for discussions about optimization algorithms. The authors would also like to thank the reviewers for their constructive remarks.

\section{REFERENCES}

[1] A. Kirsch, An Introduction to the Mathematical Theory of Inverse Problems. New York: Springer, 1996.

[2] J. A. Tropp, "Just relax: Convex programming methods for identifying sparse signals in noise," IEEE Trans. Inf. Theory, vol. 52, no. 3, pp. 1030-1051, Mar. 2006.

[3] L. I. Rudin, S. Osher, and E. Fatemi, "Nonlinear total variation based noise removal algorithms," in Proc. 11th Annu. Int. Conf. Center for Nonlinear Studies on Experimental Math.: Comput. Issues in Nonlinear Sci., Amsterdam, The Netherlands, 1992, pp. 259-268, Elsevier North-Holland, Inc.

[4] T. F. Chan, S. Osher, and J. Shen, "The digital TV filter and nonlinear denoising," IEEE Trans. Image Process., vol. 10, no. 2, pp. 231-241, Feb. 2001

[5] A. Beck and M. Teboulle, "Fast gradient-based algorithms for constrained total variation image denoising and deblurring problems," IEEE Trans. Image Process., vol. 18, no. 11, pp. 2419-2434, Nov. 2009.

[6] M. Elad, P. Milanfar, and R. Rubinstein, "Analysis versus synthesis in signal priors," Inverse Problems, vol. 23, no. 3, pp. 947-968, Jun. 2007.

[7] S. S. Chen, D. L. Donoho, and M. A. Saunders, "Atomic decomposition by basis pursuit," SIAM J. Sci. Comput., vol. 20, pp. 33-61, 1998.

[8] D. L. Donoho, "Nonlinear solution of linear inverse problems by wavelet-vaguelette decomposition," Appl. Comput. Harmon. Anal., vol. 2, pp. 101-126, 1992

[9] A. Chambolle, R. A. DeVore, N.-Y. Lee, and B. J. Lucier, "Nonlinear wavelet image processing: Variational problems, compression, and noise removal through wavelet shrinkage," IEEE Trans. Image Process., vol. 7, pp. 319-335, 1996.

[10] R. Tibshirani, "Regression shrinkage and selection via the lasso," $J$. Roy. Stat. Soc., Series B, vol. 58, pp. 267-288, 1994.

[11] C. Vonesch and M. Unser, "A fast multilevel algorithm for waveletregularized image restoration," IEEE Trans. Image Process., vol. 18, no. 3, pp. 509-523, Mar. 2009.

[12] T. Chan, S. Esedoglu, F. Park, and A. Yip, "Recent developments in total variation image restoration," in Handbook of Mathematical Models in Computer Vision. New York: Springer, 2005, pp. 17-30.

[13] W. Stefan, R. Renaut, and A. Gelb, "Improved total variation-type regularization using higher order edge detectors," SIAM J. Imag. Sci., pp. 232-251, Jan. 2010

[14] A. Chambolle and P. L. Lions, "Image recovery via total variation minimization and related problems," Numer. Math., vol. 76, pp. 167-188, 1997.

[15] J. Yuan, C. Schnörr, and G. Steidl, "Total-variation based piecewise affine regularization," in Proc. 2nd Int. Conf. Scale Space Variation. Methods Comput. Vis. (SSVM), 2009, pp. 552-564.

[16] T. F. Chan, A. Marquina, and P. Mulet, "High-order total variationbased image restoration," SIAM J. Sci. Comput., vol. 22, no. 2, pp. 503-516, 2000.

[17] T. Chan, S. Esedoglu, and S. Park, "Image decomposition combining staircase reduction and texture extraction," J. Vis. Commun. Image Rep. ,pp. 468-486, Jan. 2007.

[18] F. Knoll, K. Bredies, T. Pock, and R. Stollberger, "Second order total generalized variation (TGV) for MRI," Magn. Resonance Med., vol. 65 , no. 2, pp. $480-491,2011$.

[19] A. Chambolle, "An algorithm for total variation minimization and applications," J. Math. Imag. Vis., vol. 20, no. 1, pp. 89-97, Jan. 2004.

[20] B. Wohlberg and P. Rodriguez, "An iteratively reweighted norm algorithm for minimization of total variation functionals," IEEE Signal Proc. Lett., vol. 14, no. 12, pp. 948-951, Dec. 2007.

[21] C. R. Vogel and M. E. Oman, "Iterative methods for total variation denoising," SIAM J. Sci. Comput., vol. 17, pp. 227-238, 1996.

[22] A. Beck and M. Teboulle, "A fast iterative shrinkage-thresholding algorithm for linear inverse problems," SIAM J. Imag. Sci., vol. 2, no. 1, pp. 183-202, 2009.

[23] J. Darbon and M. Sigelle, "Image restoration with discrete constrained total variation part i: Fast and exact optimization," J. Math. Imag. Vis. vol. 26, no. 3, pp. 261-276, Dec. 2006.

[24] M. Elad, J. Starck, P. Querre, and D. Donoho, "Simultaneous cartoon and texture image inpainting using morphological component analysis (MCA)," Appl. Comput. Harmon. Anal., vol. 19, no. 3, pp. 340-358, Nov. 2005

[25] G. Steidl, J. Weickert, T. Brox, P. Mrázek, and M. Welk, "On the equivalence of soft wavelet shrinkage, total variation diffusion, total variation regularization, and sides," SIAM J. Numer. Anal., vol. 42, pp. 686-713, 2004.

[26] I. W. Selesnick and M. A. T. Figueiredo, "Signal restoration with overcomplete wavelet transforms: Comparison of analysis and synthesis priors," in Proc. SPIE, Wavelets XIII, Aug. 2-4, 2009, vol. 7446. 
[27] I. Khalidov and M. Unser, "From differential equations to the construction of new wavelet-like bases," IEEE Trans. Signal Process., vol. 54, no. 4, pp. 1256-1267, Apr. 2006.

[28] C. Vonesch, T. Blu, and M. Unser, "Generalized Daubechies wavelet families,” IEEE Trans. Signal Process., vol. 55, no. 9, pp. 4415-4429, September 2007.

[29] M. Unser and T. Blu, "Cardinal exponential splines: Part I-Theory and filtering algorithms," IEEE Trans. Signal Process., vol. 53, no. 4, pp. 1425-1438, Apr. 2005.

[30] M. Unser, "Cardinal exponential splines: Part II-Think analog, act digital," IEEE Trans. Signal Process., vol. 53, no. 4, pp. 1439-1449, Apr. 2005.

[31] A. Chambolle, "Total variation minimization and a class of binary MRF models," Lecture Notes Comput. Sci., vol. 3757, pp. 136-152, 2005.

[32] D. R. Hunter and K. Lange, "A tutorial on MM algorithms," Amer. Stat., vol. 58, no. 1, pp. 30-37, Feb. 2004.

[33] K. Lange, Optimization. New York: Springer, 2004.

[34] M. A. T. Figueiredo, J. M. Bioucas-Dias, and R. D. Nowak, "Majorization-minimization algorithms for wavelet-based image restoration," IEEE Trans. Image Process., vol. 16, no. 12, pp. 2980-2991, Dec. 2007.

[35] M. Zibulevsky and M. Elad, "L1-12 optimization in signal and image processing," IEEE Signal Process. Mag., vol. 27, no. 3, pp. 76-88, 2010.

[36] J. M. Bioucas-Dias, M. A. T. Figueiredo, and J. P. Oliveira, "Total variation-based image deconvolution: A majorization-minimization approach," in Proc. IEEE Int. Conf. Acoust., Speech, Signal Process. (ICASSP), 2006, vol. 2, p. II.

[37] J.-L. Starck, F. Murtagh, and J. M. Fadili, Sparse Image and Signal Processing: Wavelets, Curvelets, Morphological Diversity, 1st ed. Cambridge, U.K.: Cambridge Univ. Press, 2010.

[38] S. Ramani, T. Blu, and M. Unser, "Monte-Carlo SURE: A black-box optimization of regularization parameters for general denoising algorithms," IEEE Trans. Image Process., vol. 17, no. 9, pp. 1540-1554, Sep. 2008.

[39] J.-F. Aujol, G. Gilboa, T. Chan, and S. Osher, "Structure-texture image decomposition-modeling, algorithms, and parameter selection," Int. J. Comput. Vis., vol. 67, no. 1, pp. 111-136, Apr. 2006.

[40] V. Solo, "Selection of regularisation parameters for total variation denoising," in Proc. IEEE Int. Conf. Acoust., Speech, Signal Process. (ICASSP), Mar. 1999, vol. 3, pp. 1653-1655.

[41] S. D. Babacan, R. Molina, and A. K. Katsaggelos, "Parameter estimation in TV image restoration using variational distribution approximation," IEEE Trans. Image Process., vol. 17, no. 3, pp. 326-339, 2008.

[42] Y. Lin, B. Wohlberg, and H. Guo, "UPRE method for total variation parameter selection," Signal Process., vol. 90, pp. 2546-2551, Aug. 2010 .

[43] F. Jaillet and B. Torrésani, "Time-frequency jigsaw puzzle: Adaptive multiwindow and multilayered Gabor expansions," Proc. Int. J. Wavelets Multiresolution Inf. Process., vol. 5, no. 2, pp. 293-315, 2007, 05 .

[44] G. Gilboa and S. Osher, "Nonlocal operators with applications to image processing," SIAM Multiscale Model. Simul., vol. 7, no. 3, pp. 1005-1028, 2007

[45] M. Unser and P. Tafti, "Stochastic models for sparse and piecewise-smooth signals," IEEE Trans. Signal Process., vol. 59, no. 3, pp. 989-1006, Mar. 2011.

[46] T. Bengtsson and J. Cavanaugh, "An improved Akaike information criterion for state-space model selection," Comput. Stat. Data Anal. vol. 50, no. 10 , pp. 2635-2654, June 2006
[47] M. Unser and T. Blu, "Generalized smoothing splines and the optimal discretization of the Wiener filter," IEEE Trans. Signal Process., vol. 53, no. 6, pp. 2146-2159, Jun. 2005.

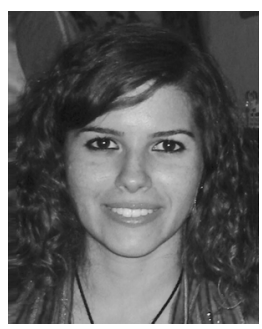

Fikret Ișık Karahanoğlu received the B.Sc. degree in electrical and electronics engineering from Middle East Technical University (METU), Turkey, in 2007 and the M.Sc. degree in communication systems from École Polytechnique Fédérale de Lausanne (EPFL), Switzerland, in 2009. She is currently working towards the Ph.D. degree at the Medical Image Processing Group affiliated to the EPFL and the University of Geneva, Switzerland.

Her research interests include inverse problems, tional magnetic resonance imaging. sparsity, regularization, and applications to func-

İker Bayram received the B.Sc. and M.Sc. degrees in electrical and electronics engineering from Middle East Technical University (METU), Ankara Turkey, and the Ph.D. degree in electrical and computer engineering from Polytechnic Institute of New York University, Brooklyn, in 2002, 2004, and 2009, respectively.

He is currently an Assistant Professor in the Department of Telecommunications and Electronics Engineering, Istanbul Technical University, Istanbul. His research interests are in wavelets, filter banks, and their applications in signal processing problems.

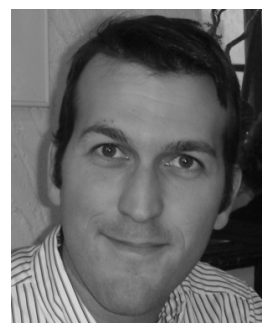

Dimitri Van De Ville (M'02) received the M.S degree in engineering and computer sciences and the Ph.D. degree from Ghent University, Belgium, in 1998, and 2002, respectively.

He received a Fellowship from the Fund for Scientific Research Flanders Belgium (FWO). In 2002, he joined Prof. M. Unser's Biomedical Imaging Group at the École Polytechnique Fédérale de Lausanne (EPFL), Switzerland. In December 2005, he became responsible for the Signal Processing Unit at the University Hospital of Geneva, Switzerland, as part of the Centre d'Imagerie Biomédicale (CIBM). In 2009, he was the recipient of a Swiss National Science Foundation professorship and currently holds a joint position at the University of Geneva, Switzerland, and the EPFL. His research interests include wavelets, sparsity, pattern recognition, and their applications in biomedical imaging, such as functional magnetic resonance imaging.

Dr. Van De Ville served as an Associate Editor for the IEEE TRANSACTIONS ON IMAGE Processing from 2006 to 2009 and the IEEE SignAl Processing LETTERS from 2004 to 2006 . He is the Vice-Chair of the Bio Imaging and Signal Processing (BISP) TC of the IEEE Signal Processing Society. Since 2003, he has also been an Editor and Webmaster of The Wavelet Digest. He is a Guest Co-Editor of the 2011 Special Issue on Brain Decoding in Pattern Recognition. $\mathrm{He}$ is Co-Chair of the 2007, 2009, and 2011 Wavelets series conferences, together with V. Goyal and M. Papadakis. 